\title{
Application of an Artificial Neural Network in Radiographic Diagnosis
}

\author{
David W. Piraino, Sundar C. Amartur, Bradford J. Richmond, Jean P. Schils, Jack M. Thome, \\ George H. Belhobek, and Mark D. Schlucter
}

\begin{abstract}
The description of 44 cases of bone tumors was used by an artificial neural network to rank the likelihood of 55 possible pathologic diagnoses. The performance of the artificial neural network was compared with the performance of experienced ( 3 or more years of radiology training) residents and inexperienced (less than 1 year of radiology training) residents. The artificial neural network was trained using descriptions of $\mathbf{1 1 0}$ radiographs of bone tumors with known diagnoses. The descriptions of a separate set of 44 cases were used to test the neural network. The neural network ranked 55 possible pathologic diagnoses on a scale from 1 to 55. Experienced and inexperienced residents also ranked the possible diagnoses in the same 44 cases. Inexperienced residents had a significantly lower mean proportion of diagnoses ranked first or second than did the neural network. Experienced residents had a significantly higher proportion of correct diagnoses ranked first than did the network. Otherwise, a significant difference between the performance of the network and experienced or inexperienced residents was not identified. These results demonstrate that artificial neural networks can be trained to classify bone tumors. Whether neural network performance in classification of bone tumors can be made accurate enough to assist radiologists in clinical practice remains an open question. These preliminary results indicate that further investigation of this technology for interpretation assistance is warranted.

Copyright 19191 by W.B. Saunders Company
\end{abstract}

KEY WORDS: neural network, diagnosis, computer, image.

\footnotetext{
RTIFICIAL neural networks (ANN) are information processing systems that automatically develop capabilities in response to information presented to them. These systems alter their information-processing capabilities according to the information input into them. ${ }^{1}$ This adaptive response is a fundamentally different approach than standard programmed

From the Departments of Radiology and Biostatistics, Cleveland Clinic Foundation, and Department of Radiology, University Hospitals of Cleveland, Case Western Reserve University, Cleveland, $\mathrm{OH}$.

Address reprint requests to Dr David W. Piraino, Diagnostic Radiology A21, Cleveland Clinic Foundation, 9500 Euclid Ave, Cleveland, OH 44195-5021.

Copyright $\cong 1991$ by W.B. Saunders Company

0897-1889/91/0404-0008\$03.00/0
}

information processing. In programmed computing, a problem is solved by devising an algorithm or set of rules that must be coded exactly into a computer program. The program can respond only according to the algorithm or set of rules in its program. ANNs do not need specific algorithms or a set of rules to solve a problem. They are trained to solve a specific problem by presenting them with examples or known solutions to the problem. They can therefore be used for problems in which specific rules or algorithms are not available.

This property of adaptive response to information makes artificial neural networks attractive for use in radiographic differential diagnosis, where specific rules or algorithms for differential diagnosis are either unknown or difficult to determine. Instead of determining a large set of specific rules or a specific algorithm to perform the radiographic diagnosis, an artificial neural network can be trained to solve this problem by presenting it with a set of cases with known diagnosis.

An artificial neural network consists of a number of simple, highly interconnected processing elements, which process information by finding the value of an output signal based on several input signals. ${ }^{2}$ After an artificial neural network has been trained, knowledge or information is stored in the way the processing elements are connected, and the way individual processing elements learn. ${ }^{3}$

Artificial neural networks are arranged in layers. Neural networks may have one or more layers. The first layer is usually the input layer, and the last layer is usually the output layer. The layers between the input and output layers are called hidden layers. A processing element has several inputs, but only one output, which is connected to many other processing elements (Fig 1). Each connection modifies the processing element's output by multiplying it by a weighting factor before inputting it into the next processing element. ${ }^{1}$ The weighting factors for each connection are independent. An artificial neural network learns or changes its response 


\section{Input layer Hidden layer Output layer}

Fig 1. Abbreviated diagram of a three-layer back propagation ANN. Diagram demonstrates the connections between the processing elements. Each element in the input layer connects to every element in the hidden layer, and each element in the hidden layer connects with every element in the output layer. Each element in the output layer has a numeric value as its output. The diseases were ranked according to the value of their corresponding output elements. $O$, processing element; $\rightarrow$, connection.

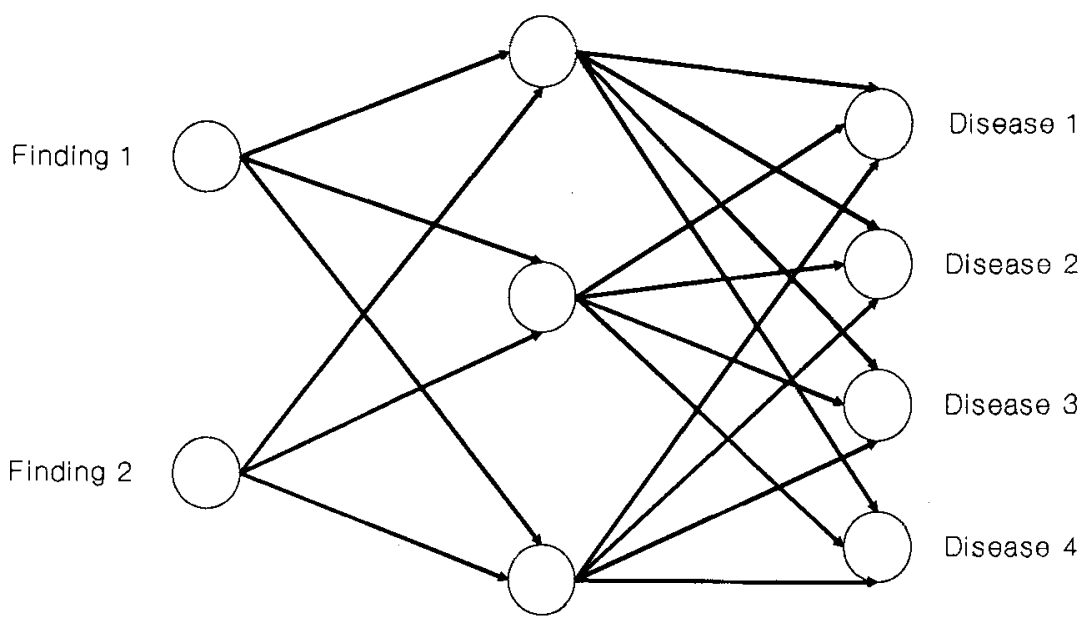

by changing the weights of the connections between processing elements. ${ }^{1}$

Each processing element has a transfer function that operates on its input to arrive at a single output. The mathematical transfer function can be one of several types, and usually restricts a processing element's output to some range of real values.

The type of neural network and its function are determined by the connections or links between elements, by the learning rules of the network, and by the processing element's inputoutput transfer function. ${ }^{4}$ A neural network learns or is trained by modifying the connection weights based on a set of training sample inputs (stimuli) and the corresponding known outputs (response). The network generates an abstraction between the inputs and outputs, enabling complex decision making in a classification task process. ${ }^{5}$

Back propagation neural networks are one type of ANN. These networks are trained to correctly classify a pattern by presenting a pattern with a known classification to the input elements.' The network produces an output based on the input pattern. This output is compared with the correct output or classification. The weights of the connections between processing elements are changed to minimize the difference between the network's output and the correct output or classification. ${ }^{1}$ This process of modifying the weights associated with the connection is propagated through the network from back to front and is called back propagation. By using multiple training examples of known classifications, the network can be trained to classify various patterns. ${ }^{1}$

The problem of producing a correct diagnosis from an image description can be considered a pattern recognition problem. The description is a symbolic pattern that must be recognized or classified as a specific disease from a known set of diseases.

Several expert systems have been applied to the problem of disease classification to assist radiologists in image interpretation. These systems have been used for consultation and diagnosis in mammography, musculoskeletal disease, lung disease, and others. ${ }^{6.8}$ Several problems with expert systems have been noted. These include difficulties with applying these systems to multiple areas in radiology, problems of differing descriptions by different individuals, knowledge representation, and maintaining the encoded knowledge. ${ }^{9}$

Neural networks have properties that could solve several of these problems. Neural networks are trained to classify patterns by presenting known cases or patterns to the network. This method allows application of the same type of neural network to multiple areas in radiographic differential diagnosis using different training cases. The problem with varying descriptions of a single case by different individuals could also be potentially solved with using neural networks by having the network trained to the descriptions provided by specific individuals. This would involve each individual describ- 
ing a training set of cases according to his or her preferences.

Knowledge representation remains a potential problem with neural networks. Knowledge representation is an inherent part of the networks, but is of a different type than that in an expert system. In neural networks, knowledge representation issues deal with problems of how to train the neural network in response to feedback and how to categorize findings, and what type of network organization is best for the specific class of problems.

This study used a back propagation, threelayer ANN to classify descriptions of bone tumors. The network's rank of the pathologic diagnosis was compared with that of experienced residents ( 3 or more years of radiology training) and inexperienced residents (less than 1 year of radiology training).

\section{METHODS}

A neural network with one hidden layer, one input layer, and one output layer was constructed. This network had 20 input elements, 40 elements in the hidden layer, and 55 elements in the output layer. Each input element represented one category of possible radiographic findings. The symbolic descriptions of bone tumors were converted to a numeric representation as demonstrated in Table 1. The numeric representation was used as the input to the input elements of the neural network. Each output element corresponded to one of the 55 possible diagnoses listed in Table 2. A back propagation learning rule was used to adjust the processing element weighting factors during training. The network was constructed using ExploreNet 3000 (HNC, San Diego, CA), which runs on an IBM PC-compatible computer.

The descriptions of 110 bone tumors with known pathologic diagnosis obtained from standard textbook illustrations were used to train the neural network. ${ }^{10}$ An experienced radiologist produced the descriptions from the textbook illustrations according to the categories in Table 1. A set of 44 bone tumors with known diagnoses obtained from the Cleveland Clinic teaching file were used as unknowns for the neural network, experienced residents, and inexperienced residents. Some residents did not read all of the films. Residents were encouraged to work through the cases in the same sequence.
The inputs into the neural network were obtained from the same radiologist who described the training set. The inexperienced residents, experienced residents, and neural network ranked the possible diagnoses from most likely to least likely with the most likely being ranked number one. The network's ranks were determined by the values of the output nodes of the network with the most likely diagnosis having the highest value. The ranks of the pathologic diagnoses were then compared using a paired $t$ test. Paired $t$ tests were used to test whether the average difference in proportions between inexperienced residents or experienced residents and the neural network differed from zero. The paired $t$ tests assume that the particular residents selected in this study represent a random sample from the population of all possible residents.

\section{RESULTS}

The comparison by rank of the pathologic diagnoses for the neural network, experienced residents, and inexperienced residents are shown in Tables 3 and 4 . When compared with the network, first-year residents had a significantly lower mean proportion of pathologic diagnoses ranked first or second than did the network (difference in mean proportions $=.165, P=$ $.021)$. Fourth-year residents had a significantly higher portion of pathologic diagnoses ranked first than did the network (difference in mean proportions $=.150, P=.002$ ). A significant difference was not identified between experienced residents and the network in percent of pathologic diagnoses ranked first or second, or in the top five.

\section{DISCUSSION}

In this study, an ANN was slightly less accurate than the group of experienced residents in rating the pathologic diagnosis as the most likely, but the neural network was slightly more accurate than the group of inexperienced residents in rating the pathologic diagnosis as one of the two most likely. These results are similar to a previous study on ANN diagnosis of 10 cases of similated interstitial lung disease where the ANN performance was comparable with chest radiologists. ${ }^{11}$ These preliminary results indicate that ANNs can be trained to classify bone tumors and perform at a level in this 
Table 1. Numeric Representation of Symbolic Findings

\begin{tabular}{|c|c|c|}
\hline $\begin{array}{l}\text { Input Neural } \\
\text { Processing } \\
\text { Element Number }\end{array}$ & Symbolic Finding & $\begin{array}{c}\text { Numeric } \\
\text { Value } \\
\text { (range, }-0.5 \\
\text { to 1.2) }\end{array}$ \\
\hline \multirow[t]{2}{*}{1} & Normal & 1.0 \\
\hline & Abnormal & 0.0 \\
\hline \multirow[t]{3}{*}{2} & Age (yr) & \\
\hline & 0 & 0.0 \\
\hline & 100 & 1.00 \\
\hline \multirow[t]{3}{*}{3} & Size of lesion $(\mathrm{cm})$ & \\
\hline & 1 & 0.1 \\
\hline & 10 & 1.0 \\
\hline \multirow[t]{3}{*}{4} & No. of lesions & \\
\hline & 1 & 0.1 \\
\hline & 10 or more & 1.0 \\
\hline \multirow[t]{28}{*}{5} & Location & \\
\hline & None & 0.0 \\
\hline & Spine & \\
\hline & Cervical & 0.10 \\
\hline & Thoracic & 0.11 \\
\hline & Lumbar & 0.12 \\
\hline & Sacrum & 0.13 \\
\hline & Coccyx & 0.14 \\
\hline & Axial & \\
\hline & Pelvis & 0.20 \\
\hline & Ribs & 0.21 \\
\hline & Scapula & 0.22 \\
\hline & Clavicle & 0.23 \\
\hline & Upper extremities & \\
\hline & Humerus & 0.30 \\
\hline & Radius & 0.31 \\
\hline & Ulna & 0.32 \\
\hline & Hand/Wrist & 0.33 \\
\hline & Lower extremities & \\
\hline & Femur & 0.40 \\
\hline & Tibia & 0.41 \\
\hline & Fibula & 0.42 \\
\hline & Patella & 0.43 \\
\hline & Foot & 0.44 \\
\hline & Head & \\
\hline & Skull & 0.50 \\
\hline & Facial bones & 0.52 \\
\hline & Multiple & 1.0 \\
\hline \multirow[t]{5}{*}{6} & Position in long axis & \\
\hline & None & 0.0 \\
\hline & Diaphysis & 0.2 \\
\hline & Metaphysis & 0.5 \\
\hline & Epiphysis & 0.7 \\
\hline \multirow[t]{7}{*}{7} & Secondary position & \\
\hline & None & 0.0 \\
\hline & Diaphysis & 0.2 \\
\hline & Metaphysis & 0.5 \\
\hline & Epiphysis & 0.7 \\
\hline & Subchondral & 1.0 \\
\hline & Soft tissue & 1.1 \\
\hline \multirow[t]{8}{*}{8} & Position in transverse plane & \\
\hline & None & 0.0 \\
\hline & Central & 0.2 \\
\hline & Eccentric & 0.3 \\
\hline & Cortical & 0.4 \\
\hline & Ajacent to cortex & 0.6 \\
\hline & Exostotic & 0.8 \\
\hline & Soft tissue & 1.0 \\
\hline
\end{tabular}

Table 1. (Cont'd)

\begin{tabular}{|c|c|c|}
\hline $\begin{array}{l}\text { Input Neural } \\
\text { Processing } \\
\text { Element Number }\end{array}$ & Symbolic Finding & $\begin{array}{l}\text { Numeric } \\
\text { Value } \\
\text { (range, }-0.5 \\
\text { to 1.2) }\end{array}$ \\
\hline \multirow[t]{4}{*}{9} & Density & \\
\hline & Normal & 0.0 \\
\hline & Lytic & 0.2 \\
\hline & Sclerotic & 1.0 \\
\hline \multirow[t]{5}{*}{10} & Tumor matrix & \\
\hline & None & 0.0 \\
\hline & Ground/glass & 0.3 \\
\hline & Calcified & 0.6 \\
\hline & Bone & 1.0 \\
\hline \multirow[t]{5}{*}{11} & Zone of transition & \\
\hline & None & 0.0 \\
\hline & Narrow & 0.2 \\
\hline & Intermediate & 0.5 \\
\hline & Wide & 1.0 \\
\hline \multirow[t]{5}{*}{12} & Aggressiveness of destruction & \\
\hline & None & 0.0 \\
\hline & Geographic & 0.2 \\
\hline & Moth-eaten & 0.7 \\
\hline & Permeative & 1.0 \\
\hline \multirow[t]{5}{*}{13} & Cortical changes & \\
\hline & None & 0.0 \\
\hline & Scalloping & 0.3 \\
\hline & Expansion & 0.7 \\
\hline & Penetration & 1.0 \\
\hline \multirow[t]{4}{*}{14} & Sclerotic margin & \\
\hline & None & 0.0 \\
\hline & Thin & 0.5 \\
\hline & Thick & 1.0 \\
\hline \multirow[t]{4}{*}{15} & Periosteal reaction & \\
\hline & None & 0.0 \\
\hline & Uninterrupted & 0.5 \\
\hline & Interrupted & 1.0 \\
\hline \multirow[t]{7}{*}{16} & Periosteal reaction & \\
\hline & None & 0.0 \\
\hline & Solid & 0.1 \\
\hline & Multiple layer & 0.2 \\
\hline & Codeman's & 0.4 \\
\hline & Sunburst & 0.6 \\
\hline & Hair-on-end & 0.8 \\
\hline \multirow[t]{6}{*}{17} & Trabeculation & \\
\hline & None & 0.0 \\
\hline & Delicate & 0.2 \\
\hline & Coarse & 0.4 \\
\hline & Lobulated & 0.6 \\
\hline & Radiating & 0.8 \\
\hline \multirow[t]{5}{*}{18} & Other findings & \\
\hline & None & 0.0 \\
\hline & Fallen fragment & 0.2 \\
\hline & Nidus & 0.4 \\
\hline & Spiculated & 0.6 \\
\hline 19 & Reserved for MR findings & \\
\hline 20 & Reserved for CT findings & \\
\hline
\end{tabular}

limited study similar to radiology residents. We believe that these results are encouraging for the use of neural networks in radiographic diagnostic assistance given that this study was 
Table 2. Diseases

\begin{tabular}{|c|c|}
\hline $\begin{array}{l}\text { Output Neural } \\
\text { Processing } \\
\text { Element Number }\end{array}$ & Disease \\
\hline 1 & Normal \\
\hline 2 & Adamantinoma \\
\hline 3 & Aneurysmal bone cyst \\
\hline 4 & Ameloblastoma \\
\hline 5 & Angiosarcoma \\
\hline 6 & Bone cyst \\
\hline 7 & Bone infarct \\
\hline 8 & Bone island \\
\hline 9 & Brodie's abscess \\
\hline 10 & Brown tumor \\
\hline 11 & Chondroblastoma \\
\hline 12 & Chondroma \\
\hline 13 & Chondromyxoid fibroma \\
\hline 14 & Chondrosarcoma \\
\hline 15 & Chordoma \\
\hline 16 & Cystic angiomatosis \\
\hline 17 & Desmoplastic fibroma \\
\hline 18 & Eosinophilic granuloma \\
\hline 19 & Epidermoid cyst \\
\hline 20 & Ewing's sarcoma \\
\hline 21 & Fibroma, nonossifying \\
\hline 22 & Fibroma, ossifying \\
\hline 23 & Fibrosarcoma \\
\hline 24 & Fibrous dysplasia \\
\hline 25 & Giant cell, nonaggressive \\
\hline 26 & Giant cell, aggressive \\
\hline 27 & Glomus turnor \\
\hline 28 & Hemangioma \\
\hline 29 & Hemangiopericytoma \\
\hline 30 & Leiomyoma \\
\hline 31 & Leiomyosarcoma \\
\hline 32 & Leukemia \\
\hline 33 & Lipoma \\
\hline 34 & Liposarcoma \\
\hline 35 & Lymphangioma \\
\hline 36 & Lymphoma \\
\hline 37 & Malignant fibrous histiocytoma \\
\hline 38 & Metastatic, Lytic \\
\hline 39 & Metastatic, blastic \\
\hline 40 & Myeloma \\
\hline 41 & Myositis ossificans \\
\hline 42 & Neurilemoma \\
\hline 43 & Neurofibroma \\
\hline 44 & Osteoblastoma \\
\hline 45 & Osteochondroma \\
\hline 46 & Osteoid osteoma \\
\hline 47 & Osteoma \\
\hline 48 & Osteomyelitis, acute \\
\hline 49 & Osteomyelitis, chronic \\
\hline 50 & Osteosarcoma \\
\hline 51 & Paget's \\
\hline 52 & Periosteal, chondroma \\
\hline 53 & Periosteal, osteosarcoma \\
\hline 54 & Periosteal desmoid \\
\hline 55 & Rhabdomyosarcoma \\
\hline
\end{tabular}

Table 3. Inexperienced Residents v Network

\begin{tabular}{|c|c|c|c|c|}
\hline $\begin{array}{l}\text { Resident } \\
\text { Number }\end{array}$ & Resident & Network & $\begin{array}{l}\text { Difference } \\
\text { Network Minus } \\
\text { Resident }\end{array}$ & $\begin{array}{l}\text { Paired } t \\
\text { PValue }\end{array}$ \\
\hline \multicolumn{5}{|c|}{ Proportion of diagnoses ranked first } \\
\hline 1 & .29 & .29 & .0 & \\
\hline 2 & .32 & .32 & .0 & \\
\hline 3 & .26 & .32 & .06 & \\
\hline 4 & .25 & .25 & .0 & \\
\hline Mean & .28 & .295 & .015 & .391 \\
\hline \multicolumn{5}{|c|}{ Proportion of diagnoses ranked first or second } \\
\hline 1 & .43 & .50 & .07 & \\
\hline 2 & .47 & .63 & .16 & \\
\hline 3 & .35 & .53 & .18 & \\
\hline 4 & .33 & .58 & .25 & \\
\hline Mean & .395 & .560 & .165 & $.021^{*}$ \\
\hline \multicolumn{5}{|c|}{ Proportion of diagnoses ranked in top five } \\
\hline 1 & .54 & .68 & .14 & \\
\hline 2 & .68 & .74 & .06 & \\
\hline 3 & .65 & .68 & .03 & \\
\hline 4 & .42 & .79 & .37 & \\
\hline Mean & .573 & .723 & .150 & .146 \\
\hline
\end{tabular}

* Statistically significant $P$ value.

an early work in this area. Further studies are needed to determine whether the performance of neural networks in the classification of radiographic abnormalities can be improved enough to be useful in clinical radiology.

There are several technical areas in which further investigation could improve the perfor-

Table 4. Experienced Residents v Network

\begin{tabular}{|c|c|c|c|c|}
\hline $\begin{array}{l}\text { Resident } \\
\text { Number }\end{array}$ & Resident & Network & $\begin{array}{l}\text { Difference } \\
\text { Network Minus } \\
\text { Resident }\end{array}$ & $\begin{array}{l}\text { Paired } \mathrm{t} \\
P \text { Value }\end{array}$ \\
\hline \multicolumn{5}{|c|}{ Proportion of diagnoses ranked first } \\
\hline 5 & .47 & .41 & -.06 & \\
\hline 6 & .53 & .37 & -.16 & \\
\hline 7 & .56 & .33 & -.23 & \\
\hline 8 & .50 & .38 & -.12 & \\
\hline 9 & .52 & .34 & -.18 & \\
\hline Mean & .516 & .366 & -.15 & $.006^{*}$ \\
\hline \multicolumn{5}{|c|}{ Proportion of diagnoses ranked first or second } \\
\hline 5 & .59 & .65 & .06 & \\
\hline 6 & .74 & .68 & -.06 & \\
\hline 7 & .56 & .61 & .05 & \\
\hline 8 & .63 & .63 & .0 & \\
\hline 9 & .59 & .52 & -.07 & \\
\hline Mean & .622 & .618 & -.004 & .889 \\
\hline \multicolumn{5}{|c|}{ Proportion of diagnoses ranked in top five } \\
\hline 5 & .65 & .76 & .11 & \\
\hline 6 & 1.0 & .79 & -.21 & \\
\hline 7 & .67 & .72 & .05 & \\
\hline 8 & .75 & .75 & .0 & \\
\hline 9 & .73 & .70 & -.03 & \\
\hline Mean & .760 & .744 & -.016 & .782 \\
\hline
\end{tabular}

* Statistically significant $P$ value. 
mance of neural networks. The network used in this study is only one type of neural network that has been applied to pattern recognition problems. The specific network used in this study, the back propagation network, is known to be slow in the training phase, and no rules are available for the optimum choice of number of hidden layers and number of nodes per layer. ${ }^{12}$ Other types of networks, including functional link networks and Hopfield networks, have been successfully used in pattern recognition problems, ${ }^{5,13}$ and their use might improve the performance of classification of radiographic abnormalities from image descriptions.

In addition, further work must be done to address the choice of training feature network elements, radiographic findings, and number of training cases that provide the optimum performance in radiographic classification. Additional training cases may provide a better representation of the possible diagnoses and might improve the network's performance.

The problem of how best to present the symbolic descriptions to the network also remains a problem area that needs further investigation. The use of a numeric representation of symbolic descriptions is only one of many possible ways to input the image description. In this type of numeric representation numerical differences within one category represent important symbolic differences in the image description. For example, the numeric difference between moth-eaten and permeative (.3) is less than the numeric difference between moth-eaten and geographic (.5), and this mapping represents symbolic relative differences between motheaten, permeative, and geographic. This type of symbolic representation can lead to meaningless pseudo-correlations. For example, the value for skull is 0.50 , for the foot is 0.44 , and for the cervical spine is 0.1 . This would mean that the foot lesion is similar to a head lesion, but different from a cervical spine lesion. These pseudo-correlations should be avoided in future representation. Fuzzy sets also could be used to represent the symbolic image description, thereby allowing the possibility of including uncertainty in the image description.

A multilayer network divides the multidimensional finding or feature space into complex regions that define the various classifications or diagnoses. The regions are defined through the training process. ${ }^{5}$ Unfortunately, it is not easy to demonstrate how different regions relate to one another. It is therefore difficult to provide an explanation of how or why a specific classification is made. Expert systems, on the other hand, provide an explanation on how and why decisions are made. Models of neural networks are being developed that can demonstrate how specific inputs affect the recognition or classification process. ${ }^{14}$ These types of networks may be able to demonstrate which findings are most critical in making a diagnosis. Hybrid systems using concepts from expert systems and ANN may prove to be the best choice in image interpretation assistance. Such systems could potentially learn by "experience" using ANNs, and could provide explanations or consultation on how decisions were made using expert systems.

Finally, studies must be conducted to test the effectiveness of neural networks in assisting radiologists in image interpretation. Will the radiologists' performance be improved with the assistance of a neural network system, or will such a system only add information confusion to an already information-overloaded radiologist?

\section{REFERENCES}

1. Hecht-Nielsen R: Neurocomputing. New York, NY, Addison-Wesley, 1990

2. Lippmann RP: An introduction to computing with neural nets. IEEE.ASSP Magazine 4:4-22, 1987

3. Engel CW, Cran M: Pattern classification: A neural network competes with humans. PC AI 4:20-62, 1990

4. Caudill M: Neural Network Primer. AI Expert. San Francisco, CA, Mills Freedman, 1989

5. Pao YH: Adaptive Pattern Recognition and Neural Networks. New York, NY, Addison-Wesley, 1989

6. de Vries PH, de Vries PF: An overview of medical expert systems. Methods Inf Med 24:57-64, 1985
7. Swett HA, Fisher PR, Cohn AI, et al: Expert systemcontrolled image display. Radiology 172:487-493, 1989

8. Lodwick GS, Haun CL, Smith WE, et al: Computer diagnosis of primary bone tumors: A preliminary report. Radiology 80:273-275, 1963

9. Piraino DW, Richmond BJ, Uetani M, et al: Problems in applying expert system technology to radiographic image interpretation. J Digital Imaging 1:21-26, 1989

10. Hudson TM: Radiologic-Pathologic Correlation of Musculoskeletal Lesions. Baltimore, MD, Williams \& Wilkins, 1987 
11. Asad N, Doi K, MacMahon H, et al: Potential usefulness of an artificial neural network for differential diagnosis of interstitial lung disease: Pilot study. Radiology 177:857-860, 1990

12. Caudill M: Neural network training tips and techniques. AI Expert 6:56-61, 1991
13. Kamgan-Parsi B, Gualtieri JA, Devaney JE, et al: Clustering with neural networks. Biol Cybern 63:201-208, 1990

14. Caudill M: Using neural nets: Hybrid expert networks. AI Expert 5:49-54, 1990 